Bull. Austral. Math. Soc.

VoL. 39 (1989) [167-170]

\title{
ON A SUBCLASS OF BAZILEVIC FUNCTIONS
}

\author{
M.M. ELHOSH
}

Integral mean and coefficient bounds for some Bazilevic functions are determined.

\section{INTRODUCTION}

Let $S$ denote the class of functions

$$
f(z)=z+\sum_{n=2}^{\infty} a_{n} z^{n}
$$

which are univalent in $|z|<1$. Let $B(\alpha)$ denote the functions which can be written in the form

$$
f(z)=\left\{\alpha \int_{0}^{z} g^{\alpha}(t) P(t) t^{-1} \mathrm{~d} t\right\}^{1 / \alpha}
$$

where $g(z)$ and $P(z)$ are subject to the conditions $g(0)=g^{\prime}(0)-1=0, \operatorname{Re} z g^{\prime}(z) / g(z)$ $\geqslant 0$ and $P(0)=1, \operatorname{Re} P(z) \geqslant 0$ respectively. Then it is well-known that $B(\alpha) \subseteq S$ $([13,6,7,8])$. The coefficients problem for $f(z)$ in $B(\alpha)$ and $S$ has been settled by Leach [7] and de Branges [1], respectively.

In this paper we study the coefficients probem for $f(z) \in B(\alpha)$ when $P(t)=1$. This type of function has been shown to be starlike in [3, Theorem 2]. We shall denote this type of function by $B_{1}(\alpha)$ and deduce some integral mean as well as coefficient bounds for the case $0<\alpha \leqslant 1$. We shall be using the notation $f \prec F$ to mean that $f(z)=F(\phi(z))$ where $\phi(z)$ satisfies $\phi(0)=0$ and $|\theta(z)| \leqslant 1$ in $|z|<1 ; f(z)$ is said to be subordinate to $F(z)([2, \mathrm{p} .190],[5, \mathrm{p} .178])$. We shall also use the notation $\sum a_{n} z^{n} \ll \sum b_{n} z^{n}$ to mean that $\left|a_{n}\right| \ll b_{n}$ for $n=1,2, \ldots$ ([5, vol. 2, Theorem 5], $[\mathbf{6}])$.

Received 19 April, 1988

Copyright Clearance Centre, Inc. Serial-fee code: 0004-9729/89 \$A2.00+0.00. 


\section{SUBORDINATION}

TheOREM 1. Let $f(z) \in B_{1}(\alpha)$. Then for $0<\alpha \leqslant 1$ we have

$$
\begin{aligned}
\log f^{\prime}(z) & \prec \log z^{-1} K(z), \\
f^{\prime}(z) & \prec z^{-1} K(z),
\end{aligned}
$$

where $K(z)=z(1-z)^{-2}$ is the well-known Koebe function.

Proof: We see from (2) that

$$
\log f^{\prime}(z)=(1-\alpha) \log z^{-1} f(z)+\alpha \log z^{-1} g(z) .
$$

Now it is well-known (see question 13 of $[2, \mathrm{p} .213]$ and $[4, \mathrm{p} .118]$ ) that

$$
\begin{aligned}
& \log z^{-1} g(z) \prec \log z^{-1} K(z), \\
& \log z^{-1} f(z) \prec \log z^{-1} K(z),
\end{aligned}
$$

since $g(z)$ and $f(z)$ are both starlike.

These, together with (3), give, since the righthandside is a convex combination of Koebe functions, that

$$
\log f^{\prime}(z) \prec \log z^{-1} K(z)
$$

as required in the first part of Theorem 1 . The second part follows by exponentiation since subordination is preserved in this case (see $[9, \mathrm{pp} .23-24]$ ).

\section{INTEGRAL MEAN BOUNDS}

THEOREM 2. Let $f(z) \in B_{1}(\alpha)$. Then for $z=r e^{i \theta}$, with $0<r<1$, we have, for $\lambda>0$, that

$$
\begin{aligned}
& \int_{0}^{2 \pi}\left|f^{\prime}(z)\right|^{\lambda} d \theta \leqslant \int_{0}^{2 \pi}\left|z^{-1} K(z)\right|^{\lambda} d \theta, \\
& \int_{0}^{2 \pi} \log \left|f^{\prime}(z)\right| d \theta \leqslant \int_{0}^{2 \pi} \log \left|z^{-1} K(z)\right| d \theta, \\
& \int_{0}^{2 \pi}\left|\log f^{\prime}(z)\right|^{\lambda} d \theta \leqslant \int_{0}^{2 \pi}\left|\log z^{-1} K(z)\right|^{\lambda} d \theta .
\end{aligned}
$$

Proof: This follows from Theorem 1 and [2, Theorem 6.1], [5, vol. 2, pp.178181].

Remark 1. Using the Bernstein $\star$-function argument (see $[2$, Chapter 7$],[8,10]$ ), we can extend the first part of Theorem 2 to negative values of $\lambda$. Also the argument of [4, Theorem 1] may be applied for $0<\lambda \leqslant 2$ in the third part of this theorem.

Remark 2. Using the coefficient formula and the first part of Theorem 2 we can see easily that $\left|a_{n}\right|<\frac{1}{2} e$ and this suggests that $\left|a_{n}\right| \leqslant 1$ for $f(z) \in B_{1}(\alpha)$, which we now prove. 


\section{Coefficient Bounds}

Theorem 3. Let $f(z) \in B_{1}(\alpha)$, let $0<\alpha \leqslant 1$, and let (1) hold. Then for $n \geqslant 1$ we have

$$
\left|a_{n}\right| \leqslant 1
$$

Proof: We see from (3) that

$$
\log f^{\prime}(z) \ll \log z^{-1} K(z)
$$

by [6, Lemma 2], since both $f(z)$ and $g(z)$ are starlike and $0<\alpha \leqslant 1$. In view of the fact that the coefficients of the righthandside of (4) are positive we deduce that

$$
f^{\prime}(z) \ll z^{-1} K(z)
$$

since exponentiation preserves majorisation in this case. This gives Theorem 3 by the definition of $\ll$ above.

The function $f(z)=z(1-z)^{-1}$ is in $B_{1}(\alpha)$ with respect to itself, since it is starlike and this shows that this bound is sharp.

\section{ODD FUNCTIONS}

Theorem 4. Let $f(z) \in B_{1}(\alpha)$, let $0<\alpha \leqslant 1$, and let $F(z)=f\left(z^{2}\right)^{\frac{1}{2}}=$ $z+a_{3} z^{3}+a_{5} z^{5}+\ldots$. Then for $n \geqslant 1$ we have

$$
\left|a_{2 n+1}\right| \leqslant \frac{1}{2 n+1}
$$

Proof: We see from (2) and [6, Lemma 2] that

$$
\begin{aligned}
F(z) & =f^{\frac{1}{2}}\left(z^{2}\right)=\int_{0}^{z} g^{\frac{1}{2}}\left(t^{2}\right) t^{-1} \mathrm{~d} t \\
& \ll \int_{0}^{z} \frac{\mathrm{d} t}{1-t^{2}} \\
& =\frac{1}{2} \log \frac{1+z}{1-z}
\end{aligned}
$$

which gives (5) by the definition of $\ll$ above.

The function $z F^{\prime}(z)=z\left(1-z^{2}\right)^{-1}$ is in $B_{1}(\alpha)$ with respect to itself since it is starlike and this shows that (5) is sharp.

Remark 3. This theorem can also be proved by the method used in the proof of Theorem 2 of $[\boldsymbol{6}]$. 


\section{REFERENCES}

[1] L. de Branges, 'A proof of the Bieberbach conjecture', Acta. Math. 154 (1985), 137-152.

[2] P.L. Duren, Univalend Functions (Springer-Verlag, New York, 1985).

[3] P.J. Eenigerburg, S.S. Miller, P.T. Mocanu and M.O. Reade, 'On a subclass of Bazilevic functions', Proc. Amer. Math. Soc. 45 (1974), 88-92.

[4] D. Girela, 'Integral means and BMOA-norms of logarithms of univalent functions', J. London Math. Soc. (2) 33 (1986), 117-132.

[5] A.W. Goodman, Univalent Functions, 2 (Mariner Pub. Co., Tampa, Florida, 1983).

[6] F.R. Keogh and S.S. Miller, 'On the coefficients of Bazilevic functions', Proc. Amer. Math. Soc. 30 (1971), 492-496.

[7] R. Leach, 'The coefficients problem for Bazilevic functions', Houston H. Math. (6) 4 (1980), 543-547.

[8] Y.J. Leung, 'Integral means of the derivatives of some univalent functions', Bull. London Math. Soc. 11 (1979), 289-294.

[8] R. Robinson, 'On the theory of univalent functions', Ann. of Math. 37 (1936), 374-408.

[10] T. Shiel-Small, 'Coefficients and integral means of some classes of analytic functions', Proc. Amer. Math. Soc. 82 (1983), 275-282.

Department of Mathematics University College of Wales, Aberystwyth, Dyfed, Wales, SY23 3BZ

United Kingdom. 